uneven training intervals outperform even intervals. In the simplified description, [PKA] rises rapidly (approximated as instantaneous) following a serotonin pulse and then decays exponentially with a time constant of 5 minutes (top, Fig. 1a). [ERK] rises and decays more slowly, described by an alpha function $\left(t e^{-\alpha t}\right)$ that peaks 45 minutes after a serotonin pulse $(1 / \alpha=45$ minutes; middle, Fig. 1a). A sequence of five evenly spaced serotonin pulses gives a peak value of the inducer (that is, the product $[\mathrm{PKA}] \times[\mathrm{ERK}])$ that is greater than that of single pulses (Fig. 1b). However, the sequence of five pulses that generates the greatest possible peak value of the inducer has uneven interpulse spacings (Fig. 1c). The optimal arrangement involves three pulses, spaced as closely together as possible, to initiate an increase in ERK activation, followed by a pause to allow the rise in ERK activity to reach its maximal value. Finally, the remaining pulses are used to increase the activity of PKA near the time of this maximum. The optimal sequence thus puts $60 \%$ of its resources into an initial burst to lift ERK activation and saves $40 \%$ to produce a final increase in PKA activity (Fig. 1c). This produces a higher peak value of the inducer than any sequence with equally spaced intervals (Fig. 1b). This simpler (and less accurate) model does not reproduce the exact interval pattern that was predicted to be optimal by Zhang et al. ${ }^{1}$, but it matches the result that uneven intervals give the largest effect.

Learning is not simply a matter of flipping biochemical switches inside neural circuits. Training sets off a cascade of genetic, biochemical and structural changes that are established over and last for a wide range of different times. The multi-timescale nature of these events accounts for the complex dynamics of forgetting. For example, curves measuring the retention of memory over time do not fall exponentially, but rather they are proportional to time raised to a negative power, so-called power-law forgetting ${ }^{11,12}$. The similarly extended timescales involved in generating synaptic modifications make the dynamics of learning equally rich. The work of Zhang et al..$^{1}$ opens up the possibility that, as we understand more about the mechanisms of neuronal circuit modification that lead to learning and begin to construct predictive models, we may be able to develop more effective, rationally designed training schedules that enhance learning.

Because elementary forms of learning, such as habituation, sensitization and classical conditioning, are extremely general, not only behaviorally but also at the molecular level, it is likely that features of stimulus sequences and reinforcement schedules are also general. The approach taken by Zhang et al. may be productive in the context of various implicit forms of learning in mammals, and this could, ultimately, lead to more effective learning protocols with clinical applications, for example to rehabilitation medicine.

\section{COMPETING FINANCIAL INTERESTS}

The authors declare no competing financial interests.

1. Zhang, Y. et al. Nat. Neurosci. 15, 294-297 (2012).

2. Castellucci, V., Pinsker, H., Kupfermann, I. \& Kandel, E.R. Science 167, 1745-1748 (1970).

3. Kandel, E.R. Science 294, 1030-1038 (2001).

4. Pinsker, H.M., Hening, W.A., Carew, T.J. \& Kandel, E.R. Science 182, 1039-1042 (1973).

5. Montarolo, P.G. et al. Science 234, 1249-1254 (1986).

6. Mauelshagen, J., Parker, G.R. \& Carew, T.J. Learn Mem. 5, 246-256 (1998).

7. Woo, N.H., Duffy, S.N., Abel, T. \& Nguyen, P.V. Hippocampus 13, 293-300 (2003).

8. Wu, G.-Y., Deisseroth, K. \& Tsien, R.W. Nat. Neurosci. 4 151-158 (2001)

9. Glenberg, A. J. Verbal Learn. Verbal Behav. 15, 1-16 (1976).

10. Cepeda, N.J., Pashler, H., Vul, E., Wixted, J.T. \& Rohrer, D. Psychol. Bull. 132, 354-380 (2006).

11. Wixted, J.T. \& Ebbesen, E. Mem. Cognit. 25, 731-739 (1997).

12. Fusi, S., Drew, P. \& Abbott, L.F. Neuron 45, 599-611 (2005).

\title{
Zooming in on seizures
}

Epilepsy affects 1-2\% of the general population and treatment options for this debilitating disorder are limited. Although the mechanisms that underlie seizure initiation and generation are still not fully understood, an imbalance between excitation and inhibition in the cortex is thought to be a critical factor. Fast-spiking interneurons that express the calcium-binding protein parvalbumin (parvalbumin-positive interneurons) make up the dominant inhibitory system of the neocortex and critically regulate the output of the pyramidal neurons. Two studies in this issue of Nature Neuroscience examine the mechanistic link between parvalbuminpositive interneurons and seizures and causally implicate parvalbumin-positive interneuron excitability to seizure generation in rodent models.

Neuregulin 1 (NRG1) is a neurotrophic factor that activates the kinase activity of ErbB4 receptors. Previous work had shown that ErbB4 expression is high in parvalbumin-positive interneurons and that NRG1 can regulate GABAergic transmission.

Li et al. on page 267 use transgenic mice, biochemistry, pharmacology and electrophysiology to manipulate NRG1-ErbB4 signaling and examine its effects on parvalbumin-positive interneuron excitability. They find that NRG1 and ErbB4 normally enhance parvalbumin-positive interneuron excitability by suppressing the voltage-activated potassium channel Kv1.1, altering the membrane threshold for the generation of action potentials. Although the detailed mechanism by which NRG1 affects Kv1.1 activity is not known, the authors find that NRG1 regulates the level of tyrosine phosphorylation of the Kv1.1 channel. Crucially, they find that mice lacking ErbB4 specifically in parvalbumin-positive interneurons have more severe seizures in response to convulsants. Li et al. also find that ErbB4, but not ErbB2, expression is reduced in cortical tissue excised from humans suffering from temporal lobe epilepsy.

In an independent report, Tan et al. on page 258 corroborate the critical involvement of NRG1-ErbB4 signaling in parvalbumin-positive interneurons to epilepsy. They find that, in a kindling model of epilepsy, seizure activity increases NRG1 levels. In the same time frame, the phosphorylated form of the ErbB4 receptor is also increased. Tan et al. also find that intracerebral infusion of NRG1 delays the onset of kindling, whereas specific deletion of ErbB4 in parvalbumin-positive interneurons promotes kindling progression. Notably, they generate mice in which ErbB4 is specifically ablated in CaMKII $\alpha$-positive neurons (presumed to be pyramidal cells) but observed no effect on seizures. Moreover, the authors report that NRG1-ErbB4 signaling affects the sprouting of hippocampal mossy fibers into the dentate inner layer, a pathological feature of limbic epilepsy.

Together, both studies provide powerful evidence to causally implicate the NR1-ErbB4 pathway in the regulation of parvalbumin-positive interneuron excitability and seizure generation, and also point to new targets for future anticonvulsive therapy.

Kalyani Narasimhan 